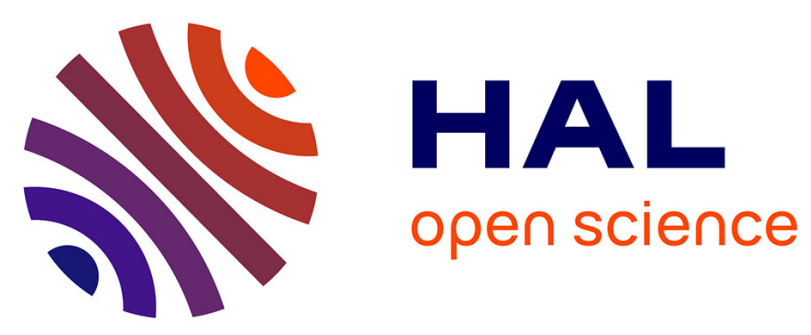

\title{
An Efficient Probabilistic Population-Based Descent for the Median Genome Problem
}

Adrien Goëffon, Macha Nikolski, David James Sherman

\section{To cite this version:}

Adrien Goëffon, Macha Nikolski, David James Sherman. An Efficient Probabilistic Population-Based Descent for the Median Genome Problem. GECCO: Genetic And Evolutionary Computation Conference, Aug 2008, Atlanta, United States. pp.315-322. hal-00341672

\section{HAL Id: hal-00341672 \\ https://hal.science/hal-00341672}

Submitted on 25 Nov 2008

HAL is a multi-disciplinary open access archive for the deposit and dissemination of scientific research documents, whether they are published or not. The documents may come from teaching and research institutions in France or abroad, or from public or private research centers.
L'archive ouverte pluridisciplinaire HAL, est destinée au dépôt et à la diffusion de documents scientifiques de niveau recherche, publiés ou non, émanant des établissements d'enseignement et de recherche français ou étrangers, des laboratoires publics ou privés. 


\title{
An Efficient Probabilistic Population-Based Descent for the Median Genome Problem
}

\author{
Adrien Goëffon, Macha Nikolski, and David Sherman \\ INRIA Bordeaux Sud-Ouest, CNRS, LaBRI \\ 351 cours de la Libération, 33400 Talence, France
}

November 25, 2008

\begin{abstract}
We present a novel population-based local search algorithm for the median genome problem. The primary result of this article is that this probabilistic approach significantly improves the performance of ancestral genome reconstruction compared to existing methods, making it possible to tackle problems where the contemporary genomes may contain many hundreds of markers. Moreover, our method is not limited to triples of genomes, and thus solves the median genome problem in its generality. We show that in real application cases the computational results are highly robust, suggesting that we can interpret the computed median genomes as candidates carrying the semantics of ancestral architectures.
\end{abstract}

\section{Introduction}

The increasing availability of fully sequenced genomes has fuelled efforts in understanding the history and function of genomes through comparison of related species and analyses in computational biology. Identifying ancestral genomes architectures is one important question that can now be addressed thanks to recent advances in combinatorial methods and to recent acquisition of large-scale genome datasets. Constructing plausible hypotheses about these ancestral architectures is a computational task whose results may provide deep insight both into the past histories of particular genomes and the 
general mechanisms of their formation. This task is frustrated by the computational complexity of the problem as well as by the difficulty of integrating biological constrains in the algorithms without altering completely their mathematical foundations.

Mathematically, genome architectures are encoded as signed permutations of common markers. The goal of this encoding is not to align one genome against the other, but rather to compare the order of gene markers. Two main approaches to compare marker orders exist: counting the differences between two genomes in terms of breakpoints [12, 15], and counting the minimal number of edit operations that transform one genome into another [8]. Both of these approaches define a distance function on the space of signed permutations. In this paper we will follow the Hannenhalli and Pevzner approach [8] where the allowed edit operations are fusion, fission, reciprocal translocation and reversal. It was originally established that this rearrangement distance can be computed in polynomial time [8, 10]. Later work has improved these results establishing a linear-time algorithm for the rearrangement distance computation [1]. Minimizing this distance is a fundamental step in computing plausible ancestors.

Two computational approaches for construction of ancestral genome architectures were proposed. They were formulated as the Median Genome Problem (MGP) and the Multiple Genome Rearrangement Problem (MGRP). Given a set of genomes $\left\{\Pi_{i}\right\}$, the former consists in computing a permutation $\Pi$ minimizing the sum of distances to $\left\{\Pi_{i}\right\}$, while the latter aims at computing the Steiner tree thus minimizing the sum of distances along its edges. The Median Genome Problem has been shown to be NP-hard for both of distance functions even in the case of only 3 genomes (see [4, 14] for breakpoint distance and [5, 6] for rearrangement distance). Nevertheless, exact resolution of MGP have been attempted, yielding optimal solutions for very small instances [17].

Approximate algorithms for MGP have been proposed for both distances. In the breakpoint case, Sankoff and Blanchette formulated the solution through a reduction to the Travelling Salesman Problem [15]. These authors proposed an algorithm that guarantees a reasonable lower bound on the sum of distances.

In the case of rearrangement distance, MGP and MGRP have been tightly linked since for real-sized cases the proposed solutions for the latter rely on successive triangulations, and thus on the solving of the former in the 3genome case. Two existing software packages MGR [3] and rEvoluzer [2] 
implement partial solutions of MGP. Indeed, MGR solves the problem for triples of multichromosomal genomes, while rEvoluzer can treat more than 3 genomes, but only in the unichromosomal case. Both of the proposed solutions are heuristics based on the detection of "good" reversals, operations that are guaranteed to improve the solution. The genomes $\left\{\Pi_{i}\right\}$ are re-written step by step by applying reversals until two (or three) of them become equal. There are two differences in the proposed solutions. First, the definition of what constitutes a good reversal is not exactly the same. Second, when no good reversals remain, MGR performs a $k$-depth search to find a best reversal, while rEvoluzer allows for backtracking.

In this paper, we present FAUCILS, a new approximate algorithm for MGP in the general case, that is, for an unrestricted number of multichromosomal genomes, while improving performances of existing approaches on restricted instances. The mainly originality of our approach is the definition of a probabilistic neighborhood which evolve within a population-based local search according to observations made on the population. This mechanism allows us to greatly accelerate the search and ensures more convergence, especially for real or structured instances.

\section{The Median Genome Problem}

A chromosome $\pi=\left(\pi_{1}, \ldots, \pi_{m}\right)$ is represented by a sequence of signed gene markers whose sign indicates their relative direction on the chromosome. A size- $n$ multichromosomal genome $\Pi$ is defined as a set of chromosomes

$\left\{\pi^{1}, \ldots, \pi^{N}\right\}$ such that $\sum_{i}\left|\pi^{i}\right|=n$. Markers take their value from the set of ordinals $1, \ldots, n$; no given marker appears in more than one chromosome.

For example, $\{(5,-8),(1,2,-10,6,4),(9),(-3,7)\}$ is a genome of size 10 . In [3], concatenation of all chromosomes is represented as a signed permutation.

Given a set of size- $n$ genomes $\left\{\Pi_{i}\right\}$ and a genome distance function $d$, an instance of the combinatorial minimization problem $M G P$ is defined by two elements $\left\langle\tau_{n}, \phi\right\rangle$ :

1. a search space, $\tau_{n}$, composed of the set of all possible size- $n$ genomes, and

2. an objective function $\phi: \tau_{n} \rightarrow \mathbb{N}($ score $)$ defined by $\phi(\Pi)=\sum_{\Pi_{i}} d\left(\Pi, \Pi_{i}\right)$. 
A median genome for a given set of genomes $\left\{\Pi_{i}\right\}$ is a genome $\Pi$ that minimizes $\phi(\Pi)$. Every optimal solution to $M G P$ is a median genome.

\section{An original population-based local search for MGP}

For addressing NP-complete problems like MGP in the general case and reaching acceptable solutions in reasonable time, approximate algorithms provide the most practical approach. We present a population-based local search algorithm using an original and evolutive neighborhood reduction mechanism for the resolution of MGP in the case of rearrangement genome distances. It gives excellent results in terms of the quality of the solutions it obtains, the speed of the computation, its robustness, and its scalability.

\subsection{A descent algorithm for MGP}

Stochastic Local Search (SLS) [9] is a well-known class of metaheuristics, used for the resolution of many difficult combinatorial optimization problems. SLS algorithms are iterative methods which start from an initial configuration (candidate solution of the search space) and improve it by successive local modifications. In this section we define a simple descent algorithm to MGP, where:

1. the initial configuration is taken from $\left\{\Pi_{i}\right\}$,

2. the evaluation function is the same as the objective function $\phi$ : the rearrangement distance $d$,

3. the neighborhood relation we call $\mathcal{R}^{1}$ is a 1 -step rearrangement: $\mathcal{R}^{1}(\Pi)=$ $\left\{\Pi^{\prime} \in \tau_{n}, d\left(\Pi, \Pi^{\prime}\right)=1\right\}$

4. the move strategy is a first-improve selection (FI) which accepts better and equivalent configurations (side-walk mechanism, SW [16]), given a specified number of iterations nbit.

The performance of a descent algorithm essentially depends on the neighborhood relation used [11]. In order to avoid slow processes and local optima 


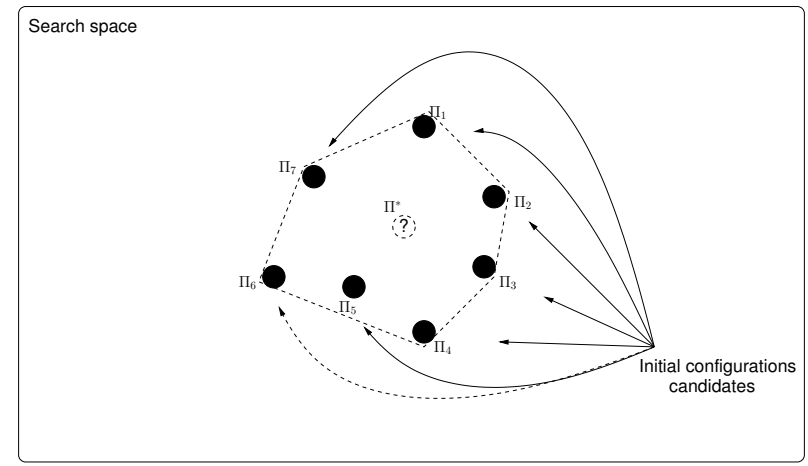

Figure 1: Geometric analogy of MGP: median genomes are within the convex hull of $\left\{\Pi_{i}\right\}$ in the space of genomes. Starting the search from a $\Pi_{i}$ on the perimeter greatly reduces the search space.

difficulties, we use the FI+SW selection strategy combined with the large and straightforward neighborhood $\mathcal{R}^{1}$.

Figure 1 shows that configurations taken from $\left\{\Pi_{i}\right\}$ may be interesting initial candidates for the beginning of the search. Considering the evaluation function and the neighborhood relation $\mathcal{R}^{1}$, the descent will explore only configurations from the schematic area delimited by the $\Pi_{i}$. If the $\Pi_{i}$ are close (for example in the case of real applications), then the resulting search space is significantly reduced.

\subsection{Probabilistic population-based local search}

Traditionally, descent algorithms are sensitive to either stochastic factors or initial configurations and consequently may not be sufficienty robust - that is, different executions may diverge - although the SW mechanism and the use of a large neighborhood can reduce this drawback. A commonly used solution is to perform several descents from different initial configurations (different replications in a multi-start descent process). In genetic local search algorithms, local search processes and crossovers between elements (individuals) of a set or multiset of current configurations (population) provide intensification and diversification phases.

A local search process applied to many independant replications is sometimes called a population-based local search even though there are no interac- 
tion between individuals [13]. Here we do not use any crossover operations, but simulate an alternative evolutionary process in order to accelerate the searches and to make multi-start descents more convergent.

We introduce a probabilistic population-based local search algorithm which favours, at each step of the search, the selection of most pertinent neighbors [7] in respect to the population. Structural information about each individual is used to estimate a selection probability at each step of the search. In this process, all replications are dependant, while the descents are carried out simultaneously.

In this section we present a multi-start descent for MGP. We use the descent mechanism presented in section 3.1, adding to each neighbor a selection probability.

Let $\mathcal{P}$ be the population of our population-based descent, which initially contains individuals taken from $\left\{\Pi_{i}\right\}$. Now let us consider a probabilistic function $p: \tau_{n} \times \tau_{n} \times \tau_{n}^{|\mathcal{P}|-1} \rightarrow[0,1]$, such that $p\left(\Pi, \Pi^{\prime}, \mathcal{P} \backslash\{\Pi\}\right)$ gives a selection probability of $\Pi^{\prime} \in \mathcal{R}^{1}(\Pi)$. Such a probabilistic function is quite similar to the one used for simulated annealing move strategy. The difference here is that only better or equivalent neighbors are accepted by the move strategy, whereas neighbors are generated by a probability distribution (probabilistic neighborhood [11]). The aim is not to escape to local optima, but to favour neighbors which share properties with other individuals in $\mathcal{P}$.

This probabilistic function is connected to the notion of adjacencies, that we define in the way analogous to Nadeau and Taylor [12]:

Definition 1 Two consecutive elements $\pi_{i}$ and $\pi_{i+1}$ of a chromosome $\pi \in \Pi$ are said to be adjacent in $\Pi$. We note this adjacency by $\left(\pi_{i} . \pi_{i+1}\right)$.

We consider additional adjacencies at the extremities of each chromosome by introducing marker 0 . For a chromosome $\left(\pi_{1}, \ldots, \pi_{m}\right)$, two adjacencies are added: $\left(0 . \pi_{1}\right)$ and $\left(\pi_{m} .0\right)$. Notice that $\left(\pi_{i} . \pi_{j}\right)=\left(-\pi_{j} .-\pi_{i}\right)$ and $\left(0 . \pi_{i}\right)=\left(-\pi_{i} .0\right)$. Finally, we note $\mathcal{A}(\Pi)$ the set of all adjacencies in $\Pi$. We have $|\mathcal{A}(\Pi)|=n+N$ ( $n$ is the number of markers and $N$ the number of chromosomes).

Each move (rearrangement) breaks one (fission) or two (reversal, fusion, reciprocal translocation) adjacencies. The probabilistic neighborhood encourages adjacencies which are not, or are less, represented in the population to be broken. The probabilistic neighbor selection operates as follows: let 
$\Pi^{\prime} \in \mathcal{R}^{1}(\Pi)$; if $\phi\left(\Pi^{\prime}\right) \leqslant \phi(\Pi)$, then $\Pi^{\prime}$ replaces $\Pi$ in $\mathcal{P}$ in function of the proportional representation of the broken adjacencies in $\mathcal{P} \backslash\{\Pi\}$ :

$$
p\left(\Pi, \Pi^{\prime}, \mathcal{P} \backslash\{\Pi\}\right)=1-\frac{\left|\left\{\Pi^{\prime \prime} \in \mathcal{P} \backslash\{\Pi\},\left(\mathcal{A}(\Pi) \backslash \mathcal{A}\left(\Pi^{\prime}\right)\right) \cap \mathcal{A}\left(\Pi^{\prime \prime}\right) \neq \emptyset\right\}\right|}{|\mathcal{P}|-1}
$$

Algorithm1 provides an overall view of our probabilistic population-based descent we called FAUCILS for Fast Ancestor (inference) Using Convergent and Intelligent Local Search.

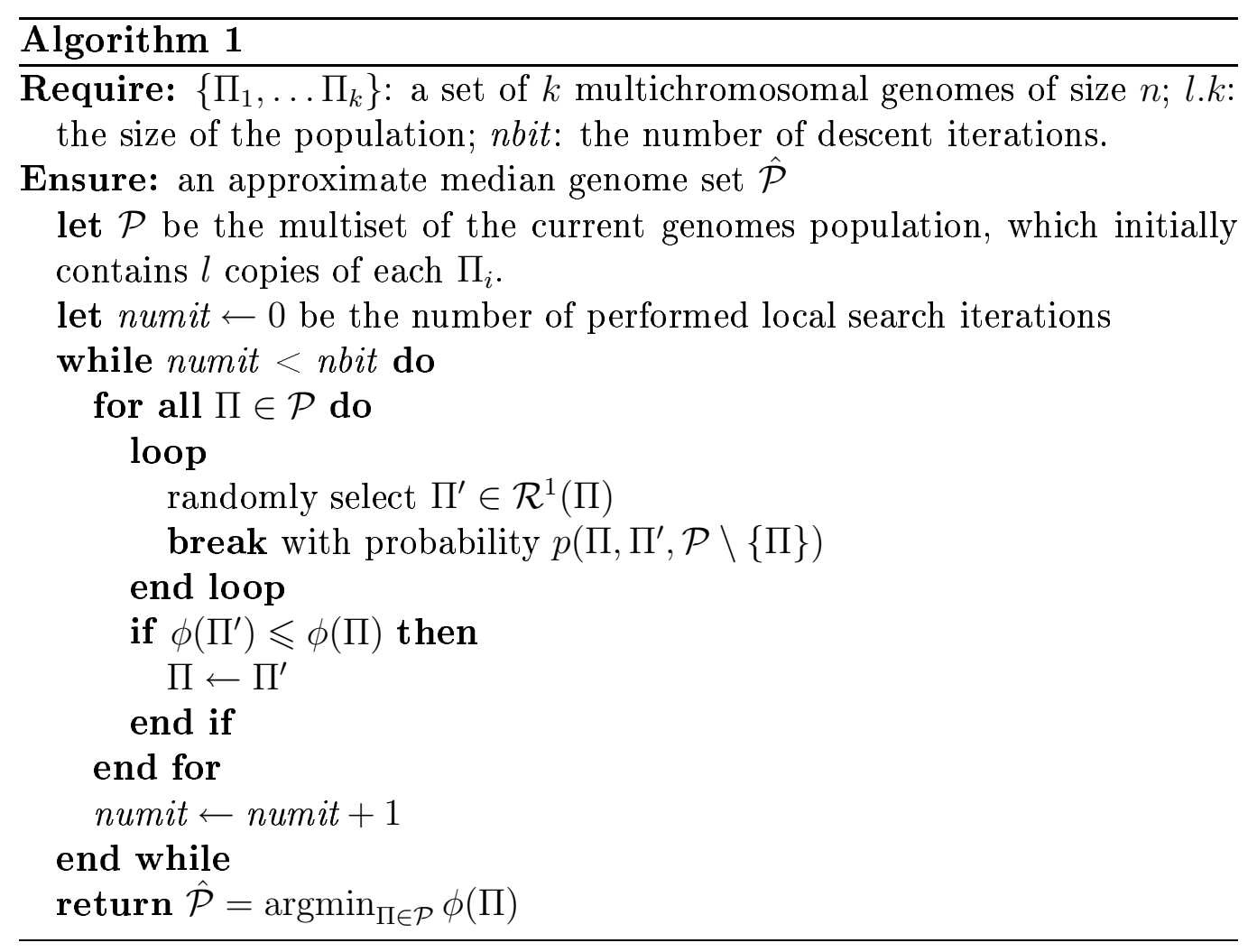

\section{Experiments}

For experiments we use different kinds of instances: real and random ones, with different numbers of genes and chromosomes by genome. 


\begin{tabular}{|c|c|c|c|c|c|c|c|c|c|c|c|c|c|}
\hline \multirow{2}{*}{ Instance } & \multirow{2}{*}{$k$} & \multirow{2}{*}{$n$} & \multicolumn{6}{|c|}{ FAUCILS } & \multicolumn{2}{|c|}{ MGR } & \multicolumn{2}{|c|}{ MGR-H1 } & \multirow{2}{*}{$\Delta$} \\
\hline & & & $\phi_{b}$ & $\mathfrak{f}$ & $\phi_{m}$ & $\phi_{w}$ & $\sigma$ & $\mathrm{CPU}$ & $\phi$ & $\mathrm{CPU}$ & $\phi$ & $\mathrm{CPU}$ & \\
\hline K135 & 5 & 135 & 281 & $7 / 20$ & 281.7 & 282 & 0.5 & $42 \mathrm{~m}$ & - & - & - & - & - \\
\hline K135-1 & 3 & 135 & 168 & $1 / 20$ & 170.6 & 172 & 1.1 & $15 \mathrm{~m}$ & 177 & $355 \mathrm{~m}$ & 178 & $44 \mathrm{~m}$ & -9 \\
\hline K135-2 & 3 & 135 & 115 & $5 / 20$ & 116.1 & 117 & 0.8 & $14 \mathrm{~m}$ & 119 & $188 \mathrm{~m}$ & 120 & $6 \mathrm{~m}$ & -4 \\
\hline K135-3 & 3 & 135 & 150 & $1 / 20$ & 151.9 & 153 & 0.7 & $15 \mathrm{~m}$ & 157 & $348 \mathrm{~m}$ & 160 & $60 \mathrm{~m}$ & -7 \\
\hline K135-4 & 3 & 135 & 132 & $14 / 20$ & 132.3 & 133 & 0.5 & $14 \mathrm{~m}$ & 135 & $377 \mathrm{~m}$ & 136 & $13 \mathrm{~m}$ & -3 \\
\hline K135-5 & 3 & 135 & 166 & $1 / 20$ & 168.0 & 169 & 0.9 & $15 \mathrm{~m}$ & 173 & $400 \mathrm{~m}$ & 175 & $48 \mathrm{~m}$ & -7 \\
\hline K135-6 & 3 & 135 & 110 & $5 / 20$ & 111.1 & 112 & 0.8 & $15 \mathrm{~m}$ & 112 & $148 \mathrm{~m}$ & 114 & $8 \mathrm{~m}$ & -2 \\
\hline K135-7 & 3 & 135 & 160 & $1 / 20$ & 161.7 & 164 & 0.9 & $13 \mathrm{~m}$ & 162 & $300 \mathrm{~m}$ & 172 & $26 \mathrm{~m}$ & -2 \\
\hline K135-8 & 3 & 135 & 185 & $4 / 20$ & 186.6 & 188 & 1.1 & $13 \mathrm{~m}$ & 193 & $527 \mathrm{~m}$ & 194 & $197 \mathrm{~m}$ & -8 \\
\hline K135-9 & 3 & 135 & 145 & $3 / 20$ & 146.6 & 148 & 1.0 & $13 \mathrm{~m}$ & 154 & $296 \mathrm{~m}$ & 154 & $45 \mathrm{~m}$ & -9 \\
\hline K135-10 & 3 & 135 & 159 & $2 / 20$ & 160.6 & 163 & 0.9 & $15 \mathrm{~m}$ & 167 & $355 \mathrm{~m}$ & 165 & $30 \mathrm{~m}$ & -6 \\
\hline K499 & 5 & 499 & 564 & $6 / 20$ & 565.9 & 568 & 1.7 & $98 \mathrm{~m}$ & - & - & - & - & - \\
\hline K499-1 & 3 & 499 & 407 & $5 / 20$ & 408.1 & 410 & 0.9 & $44 \mathrm{~m}$ & $T$ & $\max$ & 413 & $457 \mathrm{~m}$ & -6 \\
\hline K499-2 & 3 & 499 & 234 & $1 / 20$ & 235.0 & 236 & 0.3 & $38 \mathrm{~m}$ & / & $\max$ & 233 & $105 \mathrm{~m}$ & +1 \\
\hline K499-3 & 3 & 499 & 338 & $6 / 20$ & 339.1 & 341 & 0.9 & $43 \mathrm{~m}$ & / & $\max$ & 339 & $297 \mathrm{~m}$ & -1 \\
\hline K499-4 & 3 & 499 & 263 & $4 / 20$ & 263.9 & 265 & 0.6 & $39 \mathrm{~m}$ & / & $\max$ & 262 & $106 \mathrm{~m}$ & +1 \\
\hline K499-5 & 3 & 499 & 372 & $2 / 20$ & 373.7 & 375 & 1.0 & $43 \mathrm{~m}$ & / & $\max$ & 375 & $391 \mathrm{~m}$ & -3 \\
\hline К499-6 & 3 & 499 & 181 & $10 / 20$ & 181.6 & 183 & 0.6 & $38 \mathrm{~m}$ & 179 & 2 days & 179 & $74 \mathrm{~m}$ & +2 \\
\hline K499-7 & 3 & 499 & 375 & $1 / 20$ & 377.1 & 379 & 1.0 & $37 \mathrm{~m}$ & / & $\max$ & 381 & $317 \mathrm{~m}$ & -6 \\
\hline K499-8 & 3 & 499 & 476 & $3 / 20$ & 479.2 & 481 & 1.6 & $40 \mathrm{~m}$ & / & $\max$ & 484 & $823 \mathrm{~m}$ & -5 \\
\hline K499-9 & 3 & 499 & 307 & $2 / 20$ & 310.3 & 310 & 0.9 & $35 \mathrm{~m}$ & / & $\max$ & 309 & $230 \mathrm{~m}$ & -2 \\
\hline K499-10 & 3 & 499 & 338 & $3 / 20$ & 340.0 & 343 & 1.4 & $42 \mathrm{~m}$ & 1 & $\max$ & 338 & $251 \mathrm{~m}$ & $=$ \\
\hline $\mathrm{HCM}$ & 3 & 114 & 48 & $20 / 20$ & 48.0 & 48 & 0 & $<1 \mathrm{~m}$ & 48 & $10 \mathrm{~m}$ & 48 & $<1 \mathrm{~m}$ & 0 \\
\hline
\end{tabular}

Table 1: Comparison between FAUCILS and MGR on real instances. $\phi_{b}$ is the best score returned by FAUCILS, $\mathfrak{f}$ is its frequency, $\phi_{m}$ is the mean score, $\phi_{w}$ is the worst score, $\sigma$ is the standard deviation based on 20 different executions.

\subsection{Real instances}

First we assess our algorithm FAUCILS on two sets of 10 triplets of yeast genomes. The data, provided by Génolevures Consortium 1 (GDR CNRS 2354), consists in five sequenced yeasts from the Kluyveromyces clade: Kluyveromyces lactis (Klla), Saccharomyces kluyveri (Sakl), Zygosaccharomyces rouxii (Zyro), Ashbya gossypii (Ergo) and Kluyveromyces thermotolerans (Klth). From these data, two sets of permutations have been computed: the first one with 135 markers (K135), and the second one with 499 markers (K499). For the comparison with MGR, which calculates only 3-genomes medians $(N=3)$, we separate in ten instances each possible triplet of genomes: Klla-Sakl-Zyro is K135-1 and K499-1, Klla-Sakl-Ergo is K135-2 and K499-2, ... These five genomes have respectively $6,8,7,6$ and 8 chromosomes.

\footnotetext{
1 http://www.genolevures.org
} 
We add a real test instance composed by the genomes of Human, Cat and Mouse, and available on the MGR web page 2 .

Table1 show performances of FAUCILS and MGR on these real instances. FAUCILS is a stochastic algorithm, and two executions may return different results ; for each instance we perform executions. Table 1 indicates the best results $\phi_{b}$ of 20 executions, their frequency $\mathfrak{f}$, the mean scores $\phi_{m}$, the worst scores $\phi_{w}$, the standard deviations $\sigma$ and the mean computation times of one execution. FAUCILS was run with its default parameters: $l=3$ (i.e. a population size of 9 when $k=3$ ), and one million LS iterations (nbit); MGR was first run with its default parameters, and secondly with the heuristic option H1 (MGR-H1) for speeding up the search. Each execution was performed on a node of Grid' $5000^{3}$ and the computational time limit per compute node was fixed to one week. In all tables, $\Delta$ gives the difference between the best score returned by MGR and the best score returned by FAUCILS.

From Table1 1 one observes that FAUCILS computes better median genome than MGR. For all the K135 instances, FAUCILS performs better MGR with 5.9 rearrangements less per instance for the bests runs, and 4.4 rearrangements less in the mean for all the 200 runs $(10 \times 20)$ with low computation times (about 15 minutes against hours for MGR). The MGR H1 heuristic does speed up the program, but the returned solutions are less competitive (except for K135-10 where MGR-H1 beats MGR with default settings). When the number of markers is big (499), MGR needs to be used with its speed resolution heuristic to complete the search, and for these instances FAUCILS and MGR are more comparable in term of scores, although FAUCILS remains better in mean and faster.

Finally, FAUCILS is also robust with more than three genomes (instances K135 and K499) since the returned solutions have very close scores. The increase of the computation time mainly depends on the population size parameter, which can be reduced.

\subsection{Random instances}

In order to assess the performance of our population-based local search algorithm in respect of the structure and the size of the instance, we generate

\footnotetext{
${ }^{2}$ http://nbcr.sdsc.edu/GRIMM/mgr.cgi

${ }^{3}$ https://www.grid5000.fr
} 


\begin{tabular}{|c|c|c|c|c|c|c|c|c|c|c|c|c|c|}
\hline \multirow{2}{*}{ Instance } & \multirow{2}{*}{$n$} & \multirow{2}{*}{$N$} & \multicolumn{6}{|c|}{ FAUCILS } & \multicolumn{2}{|c|}{ MGR } & \multicolumn{2}{|c|}{ MGR-H1 } & \multirow{2}{*}{$\Delta$} \\
\hline & & & $\phi_{b}$ & $\mathfrak{f}$ & $\phi_{m}$ & $\phi_{w}$ & $\sigma$ & $\mathrm{CPU}$ & $\phi$ & $\mathrm{CPU}$ & $\phi$ & $\mathrm{CPU}$ & \\
\hline $\mathrm{R} 50-1$ & 50 & 1 & 79 & $2 / 20$ & 80.6 & 82 & 0.8 & $4 \mathrm{~m}$ & 82 & $6 \mathrm{~m}$ & 83 & $2 \mathrm{~m}$ & -3 \\
\hline R50-2 & 50 & $1-9$ & 80 & $1 / 20$ & 81.5 & 82 & 0.6 & $6 \mathrm{~m}$ & 87 & $5 \mathrm{~m}$ & 87 & $2 \mathrm{~m}$ & -7 \\
\hline R50-3 & 50 & $3-7$ & 80 & $6 / 20$ & 80.8 & 82 & 0.6 & $6 \mathrm{~m}$ & 84 & $7 \mathrm{~m}$ & 85 & $1 \mathrm{~m}$ & -4 \\
\hline $\mathrm{R} 50-4$ & 50 & $5-5$ & 79 & $5 / 20$ & 80.2 & 81 & 0.8 & $6 \mathrm{~m}$ & 83 & $8 \mathrm{~m}$ & 84 & $1 \mathrm{~m}$ & -4 \\
\hline R100-1 & 100 & 1 & 171 & $1 / 20$ & 173 & 175 & 1.0 & $8 \mathrm{~m}$ & 175 & $801 \mathrm{~m}$ & 177 & $126 \mathrm{~m}$ & -4 \\
\hline R100-2 & 100 & $2-10$ & 166 & $1 / 20$ & 168.8 & 170 & 1.1 & $10 \mathrm{~m}$ & 176 & $250 \mathrm{~m}$ & 178 & $226 \mathrm{~m}$ & -10 \\
\hline R100-3 & 100 & $3-7$ & 170 & $2 / 20$ & 171.9 & 174 & 1.2 & $10 \mathrm{~m}$ & 174 & $310 \mathrm{~m}$ & 179 & $139 \mathrm{~m}$ & -4 \\
\hline R100-4 & 100 & $5-5$ & 166 & $1 / 20$ & 169.8 & 172 & 1.5 & $10 \mathrm{~m}$ & 169 & $464 \mathrm{~m}$ & 171 & $170 \mathrm{~m}$ & -3 \\
\hline R200-1 & 200 & 1 & 354 & $1 / 20$ & 357.2 & 362 & 1.9 & $16 \mathrm{~m}$ & $/$ & $\max$ & 1 & $\max$ & - \\
\hline R200-3 & 200 & 10 & 351 & $1 / 20$ & 356.1 & 360 & 2.0 & $19 \mathrm{~m}$ & / & $\max$ & / & $\max$ & - \\
\hline R200-4 & 200 & $11-20$ & 344 & $2 / 20$ & 347 & 350 & 1.7 & $21 \mathrm{~m}$ & 1 & $\max$ & 366 & 4 days & -22 \\
\hline R500-1 & 500 & 1 & 924 & $1 / 20$ & 928.2 & 932 & 2.1 & $37 \mathrm{~m}$ & $T$ & $\max$ & 1 & $\max$ & - \\
\hline R500-2 & 500 & 10 & 942 & $2 / 20$ & 945.4 & 950 & 2.3 & $42 \mathrm{~m}$ & / & $\max$ & / & $\max$ & - \\
\hline R $500-2$ & 500 & $18-44$ & 936 & $1 / 20$ & 942.7 & 947 & 3.1 & $40 \mathrm{~m}$ & l & $\max$ & 1 & $\max$ & - \\
\hline
\end{tabular}

Table 2: Comparison between FAUCILS and MGR on random instances

\begin{tabular}{|c|c|c|c|c|c|c|c|c|c|c|c|c|c|c|}
\hline \multirow{2}{*}{ Instance } & \multirow{2}{*}{$n$} & \multirow{2}{*}{$N$} & \multirow{2}{*}{$\operatorname{div}$} & \multicolumn{6}{|c|}{ FAUCILS } & \multicolumn{2}{|c|}{ MGR } & \multicolumn{2}{|c|}{ MGR-H1 } & \multirow{2}{*}{$\Delta$} \\
\hline & & & & $\overline{\phi_{b}}$ & $\mathfrak{f}$ & $\phi_{m}$ & $\phi_{w}$ & $\sigma$ & CPU & $\phi$ & $\mathrm{CPU}$ & $\phi$ & $\mathrm{CPU}$ & \\
\hline S100-10-1 & 100 & 1 & 10 & 10 & $20 / 20$ & 10.0 & 10 & 0 & $<1 \mathrm{~m}$ & 10 & $<1 \mathrm{~m}$ & 10 & $<1 \mathrm{~m}$ & $=$ \\
\hline $\mathrm{S} 100-10-2$ & 100 & 5 & 10 & 10 & $20 / 20$ & 10.0 & 10 & 0 & $<1 \mathrm{~m}$ & 10 & $<1 \mathrm{~m}$ & 10 & $<1 \mathrm{~m}$ & $=$ \\
\hline S100-10-3 & 100 & 10 & 10 & 10 & $20 / 20$ & 10.0 & 10 & 0 & $<1 \mathrm{~m}$ & 10 & $<1 \mathrm{~m}$ & 10 & $<1 \mathrm{~m}$ & $=$ \\
\hline S100-50-1 & 100 & 1 & 50 & 50 & $20 / 20$ & 50.0 & 50 & 0 & $<1 \mathrm{~m}$ & 50 & $8 \mathrm{~m}$ & 51 & $<1 \mathrm{~m}$ & $\overline{=}$ \\
\hline S100-50-2 & 100 & 5 & 50 & 49 & $20 / 20$ & 49.0 & 49 & 0 & $<1 \mathrm{~m}$ & 49 & $8 \mathrm{~m}$ & 49 & $<1 \mathrm{~m}$ & $=$ \\
\hline $\mathrm{S} 100-50-3$ & 100 & 10 & 50 & 49 & $20 / 20$ & 49.0 & 49 & 0 & $<1 \mathrm{~m}$ & 49 & $13 \mathrm{~m}$ & 49 & $1 \mathrm{~m}$ & $=$ \\
\hline S100-100-1 & 100 & 1 & 100 & 95 & $7 / 20$ & 95.7 & 97 & $\overline{0.6}$ & $<1 \mathrm{~m}$ & 97 & $190 \mathrm{~m}$ & 98 & $2 \mathrm{~m}$ & $\overline{-2}$ \\
\hline S100-100-2 & 100 & 5 & 100 & 95 & $2 / 20$ & 96.4 & 98 & 0.7 & $<1 \mathrm{~m}$ & 96 & $55 \mathrm{~m}$ & 98 & $2 \mathrm{~m}$ & -1 \\
\hline S100-100-3 & 100 & 10 & 100 & 96 & $4 / 20$ & 96.9 & 98 & 0.5 & $<1 \mathrm{~m}$ & 99 & $70 \mathrm{~m}$ & 99 & $4 \mathrm{~m}$ & -3 \\
\hline S100-200-1 & $\overline{100}$ & $\overline{1}$ & 200 & 155 & $1 / 20$ & 158.6 & 160 & 1.4 & $5 \mathrm{~m}$ & 163 & $978 \mathrm{~m}$ & 166 & $65 \mathrm{~m}$ & -8 \\
\hline $\mathrm{S} 100-200-2$ & 100 & 5 & 200 & 145 & $1 / 20$ & 146.6 & 148 & 0.7 & $5 \mathrm{~m}$ & 151 & $331 \mathrm{~m}$ & 151 & $67 \mathrm{~m}$ & -6 \\
\hline $\mathrm{S} 100-200-3$ & 100 & 10 & 200 & 143 & $1 / 20$ & 145.7 & 154 & 2.2 & $5 \mathrm{~m}$ & 150 & $114 \mathrm{~m}$ & 154 & $78 \mathrm{~m}$ & -7 \\
\hline
\end{tabular}

Table 3: Comparison between FAUCILS and MGR on simulated instances

two types of random instances.

First, we use completely random instances $(R)$ containing a specified number of markers, and a minimum and maximum number of chromosomes by genome $(N)$.

On these instances of size 50 and 100, FAUCILS obtains better results than MGR systematically (see Table 2). For larger instances, only one MGR run ended, with an uncompetitive result $(\Delta=-22)$. These instances seem to be difficult because of their structure: each genome is a random point of $\tau_{n}$, and the MGR algorithm seems very dependant on the structure of each instance (see the divergences between all computational times on tables 1, 2 
and $[3$ ).

In order to estimate the impact of the structure of the instance, we generate simulated instances (S), which distances between genomes are bounded. An arbitrary ancestral genome is generated from which a specified number of random rearrangements are applied to give three genomes. We specify the number of genes $(n)$ and chromosomes $(N)$, and the number of rearrangements done during the simulation $(r)$; this parameter is an upper bound of the optimal median genome score.

The results are given in table 3. We can see that, with $r=10$ or $r=50$, instances are very easy to solve. But when the distances between genomes increase $(r=100$ and $r=200)$, FAUCILS is very competitive and can find in short computation time solutions considerably better than MGR. Moreover, the algorithm is robust as small values of $\sigma$ show. For these instances $(\mathrm{S})$, we have to reduce the number of local search iterations to 2000.r for an equivalent efficiency.

The evolution of the ratio $\phi / r$ gives an empirical indication of the structure of the search space. Indeed, for $r=200$, the minimal number of rearrangements required for reconstructing an evolutionary scenario is about $25 \%$ lower than to the number of rearrangements made during the simula-

tion. Adding to the relative difficulty to find near-optimal genomes for these instances, we can presume that this ratio represents the quantity of lost information and can be a good indicator for comparing the difficulty of simulated instances.

Finally, we have executed rEvoluzer [2] on each unichromosomal instance: S100-10-1, S100-50-1, S100-100-1, S100-200-1, R50-1, R100-1, R200-1, R-5001. Except for the three first instances, where rEvolzer found in few seconds or minutes the sames scores as FAUCILS $(10,50,95)$, the program did not returned any solution for the five other instances, even given one week of computation.

\subsection{Influence of the probabilistic neighborhood}

One of the main originalities of FAUCILS is that neighbors are selected with a non-uniform probability. The foremost aim is to select more pertinent neighbors as a function of the similarities between individuals in the current population. Since the population is initialized by the given genomes (instance), the probabilistic selection will have a larger impact on structured instances, that is when genomes shared adjacencies; it is notably the case of 
K135

best individual

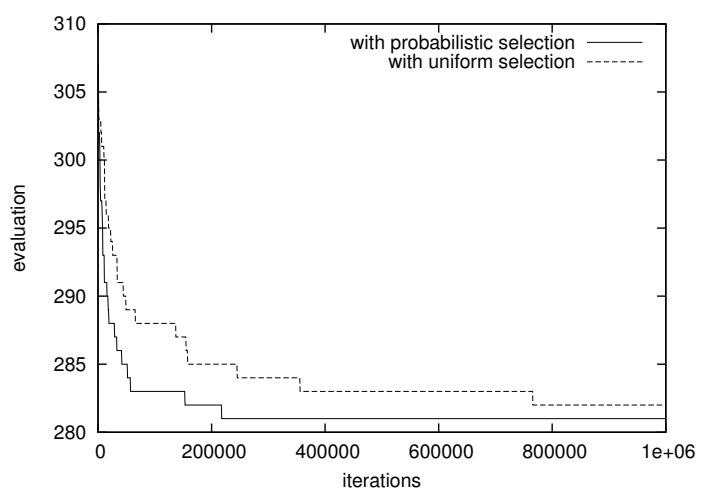

K499

best individual

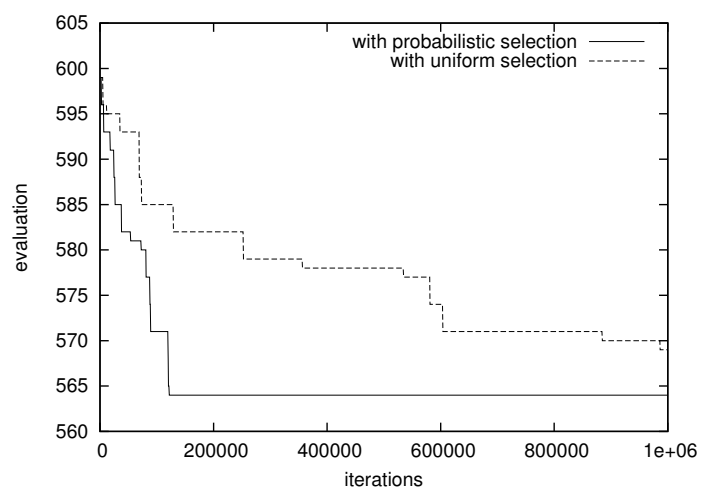

entire population

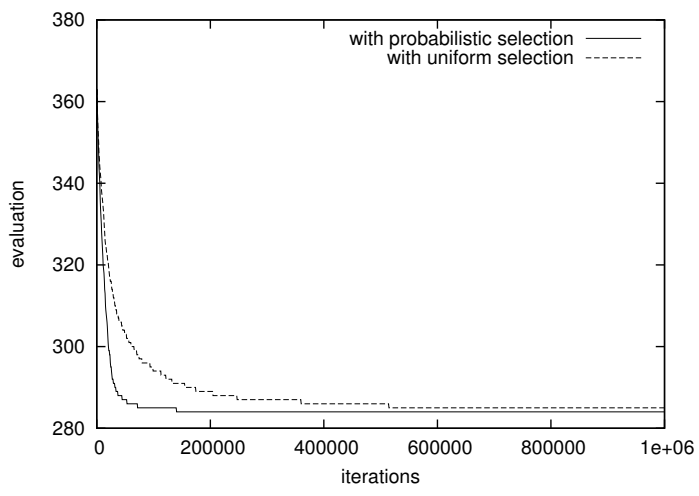

entire population

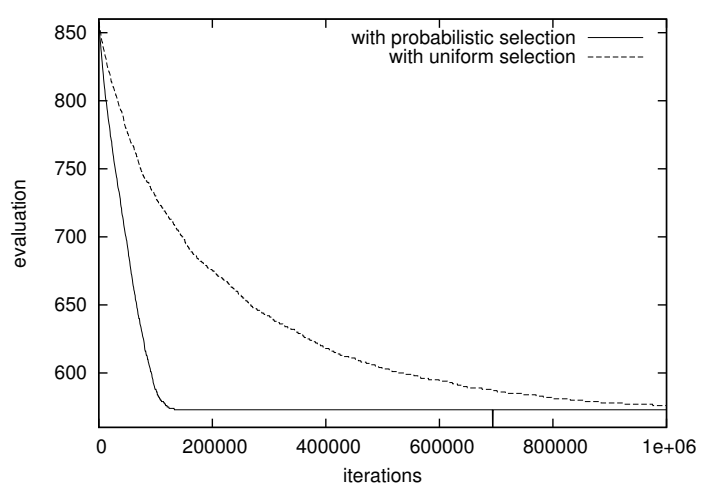

Figure 2: Influence of the probabilistic neighbor selection on real instances: evolution of the minimum and average population score during the search

real data instances.

Figure 2 shows the evolution of the minimum score (in the left) and the average score of all individuals (in the right) during the search for the complete Kluyveromyces genomes instances (K135 at the top, K499 at the bottom). We compare the probabilistic descent (algorithm 1) to the same search without a probabilistic selection (in this case each $p\left(\Pi, \Pi^{\prime}, \mathcal{P} \backslash\{\Pi\}\right)=1$, the search is a simple multi-start FI+SW descent). On both instances the probabilistic selection, which one can view as a dynamic neighborhood reduction, allows the population to converge very quickly without efficiency loss. While the 
best individual

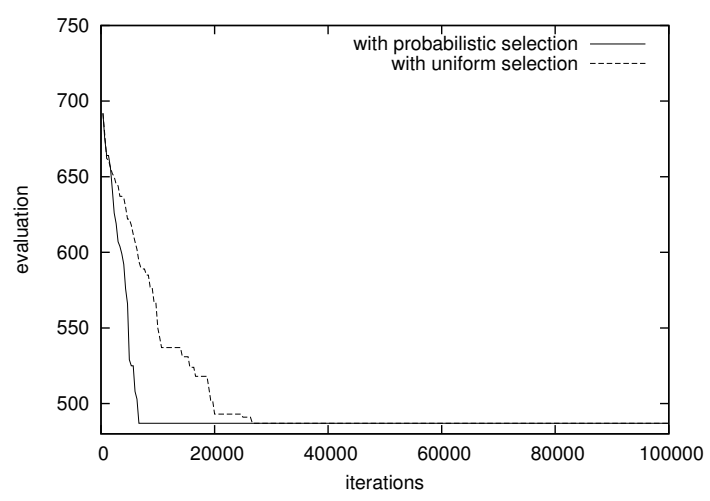

entire population

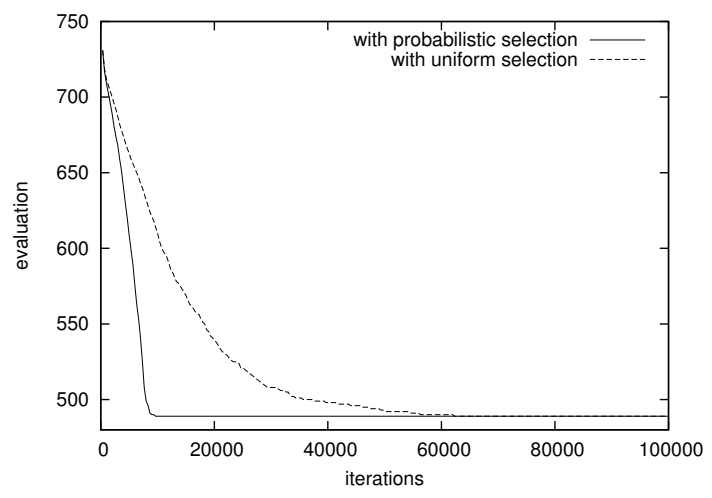

Figure 3: Influence of the probabilistic neighbor selection on simulated instances

classic search does not stabilize even after one million iterations, the probabilistic one reaches a point of stagnancy after about 200000 LS iterations. The efficiency is more significant with the largest instance (499 markers). Indeed the neighborhood is very large in this case, and such a reduction is more effective. Moreover, the augmentation of the number of markers for the same genomes significantly increases the shared adjacencies and consequently the number of non-selected neighbors.

In Figure 3, one observes a similar convergence on a simulated multigenome instance: 10 genomes obtained after 500 random rearrangements from an initial genome of size 100. On this easier instance, less than 10000 LS iterations (a few seconds of computation) are necessary to converge to supposed optimal solutions.

At the contrary, on completely random instances (figure 4), both mechanisms have the same efficiency. Indeed, such instances have insignificant numbers of shared adjacencies, and the probabilistic selection has no effect. This random instance has the same size than the simulated one (used in figure 3). We show only the average scores evolution, because for this instance the minimum scores can differ execution to execution independently from the mechanism used.

This study show that this probabilistic population-based local search add semantics to the search in order to reduce the neighborhood. It takes advantage to the structure of the instance for a quick convergence of the population. 


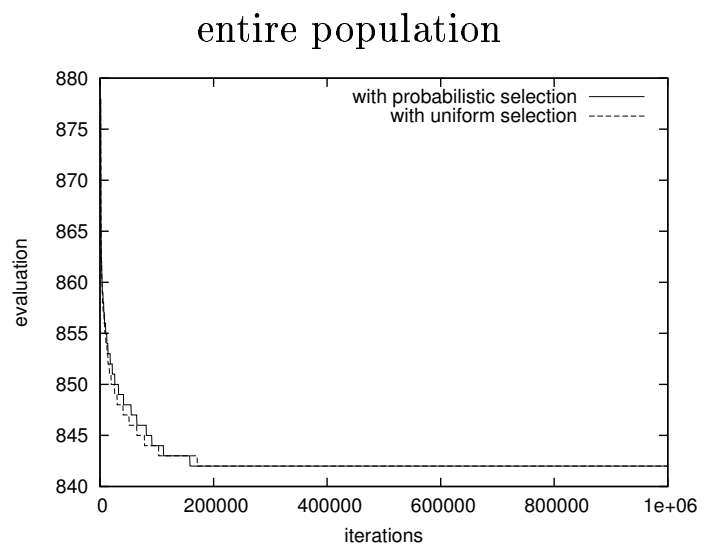

Figure 4: Probabilistic neighborhood has no effet on totally random instances

\section{Conclusion}

In this paper we propose a new efficient algorithm for the resolution of the Median Genome Problem (MGP) in the general case. We have notably introduced an novel way for speeding up and making more convergent multi-start descents for the resolution of MGP, especially for real structured instances. The key idea is to use a probabilistic neighborhood which evolves during the search according to the partial results of all descents performed simultaneously.

Experiments realized both on real and random instances show that our software FAUCILS is able to find largely better solutions than MGR, the current reference in the domain. Moreover, this local search approach is very fast and scalable: contrary to other existing techniques, FAUCILS can treat an unbounded number of multichromosomal genomes, which may contain hundred or thousands of markers. Future work will involve finding ways to evaluate the quality of solutions in the case of big instances, and to extend this MGP algorithm for the resolution of MGRP. The Median Genome Rearrangement Problem is a very hard computational problem of which existing algorithms calculate multiple median genomes for its resolution. 


\section{Acknowledgments}

We would like to thank Guillaume Bourque and Matthias Bernt for their helpful comments and for providing us their software MGR and rEvoluzer. We thank Géraldine Jean for the construction of the real signed permutations (Kluyveromyces genomes), and the Genolevures Consortium for making these data available.

Experiments presented in this paper were carried out using the Grid'5000 experimental testbed, an initiative from the French Ministry of Research through the ACI GRID incentive action, INRIA, CNRS and RENATER and other contributing partners (see https://www.grid5000.fr).

\section{References}

[1] D. A. Bader, B. Moret, and M. Yan. A linear-time algorithm for computing inversion distances between signed permutations with an experimental study. Journal of Computational Biology, 8(5):483-491, 2001.

[2] M. Bernt, D. Merkle, and M. Middendorf. Genome rearrangement based on reversals that preserve conserved intervals. IEEE/ACM Transactions on Computational Biology and Bioinformatics, 3(3):275-288, 2006.

[3] G. Bourque and P. A. Pevzner. Genome-scale evolution: Reconstructing gene orders in the ancestral species. Genome Research, 12:26-36, 2002.

[4] D. Bryant. The complexity of the breakpoint median problem. Technical Report CRM2579, Centre de Recherches Mathematiques, Universite de Montreal, 1998.

[5] A. Caprara. Formulations and complexity of multiple sorting by reversals. In S. Istrail, P. Pevzner, and M. Waterman, editors, Proceedings of the Third Annual International Conference on Computational Molecular Biology (RECOMB-99), pages 84-93, Lyon, France, 1999. ACM Press.

[6] A. Caprara. The Reversal Median Problem. INFORMS J. on Computing, 15(1):93-113, 2003.

[7] A. Goëffon, J.-M. Richer, and J.-K. Hao. Progressive tree neighborhood applied to the maximum parsimony problem. IEEE/ACM Transactions on Computational Biology and Bioinformatics, 5(1):136-145, 2008. 
[8] S. Hannenhalli and P. Pevzner. Transforming cabbage into turnip: polynomial algorithm for sorting signed permutations by reversals. In Proceedings of the 27th Annual ACM Symposium on the Theory of Computing, pages 178-189. ACM Press, 1995.

[9] H. H. Hoos and T. Stützle. Stochastic Local Search : Foundations 8 Applications (The Morgan Kaufmann Series in Artificial Intelligence). Morgan Kaufmann, 2004.

[10] J. Meidanis and J. C. Setubal. Introduction to Computational Molecular Biology. PWS Publishing, 1997.

[11] H. Muhlenbein and J. Zimmermann. Size of neighborhood more important than temperature for stochastic local search. In C. Cotta and J. I. van Hemert, editors, Proceedings of the 2000 Congress on Evolutionary Computation, volume 2, pages 1017-1024, 2000.

[12] J. Nadeau and B. Taylor. Lengths of Chromosomal Segments Conserved since Divergence of Man and Mouse. Proceedings of the National Academy of Sciences of the United States of America, Part 1: Biological Sciences, 81(3):814-818, 1984.

[13] J. M. Pasia, K. F. Doerner, R. F. Hartl, and M. Reimann. A populationbased local search for solving a bi-objective vehicle routing problem. In C. Cotta and J. I. van Hemert, editors, EvoCOP, volume 4446 of Lecture Notes in Computer Science, pages 166-175. Springer, 2007.

[14] I. Pe'er and R. Shamir. The median problems for breakpoints are NP-complete. Electronic Colloquium on Computational Complexity (ECCC), 5(071), 1998.

[15] D. Sankoff and M. Blanchette. The median problem for breakpoints in comparative genomics. In COCOON '97: Proceedings of the Third Annual International Conference on Computing and Combinatorics, pages 251-264, London, UK, 1997. Springer-Verlag.

[16] B. Selman, H. Levesque, and D. Mitchell. A new method for solving hard satisfiability problems. In Proceedings of the Tenth National Conference on Artificial Intelligence (AAAI-92), pages 440-446, San Jose, CA, USA, 1992. 
[17] A. C. Siepel and B. M. E. Moret. Finding an optimal inversion median: Experimental results. In WABI '01: Proceedings of the First International Workshop on Algorithms in Bioinformatics, pages 189-203, London, UK, 2001. Springer-Verlag. 